\title{
(6) OPEN ACCESS \\ Optical coherence tomography in the preoperative and postoperative management of macular hole and epiretinal membrane
}

\author{
Roger A Goldberg, Nadia K Waheed, Jay S Duker
}

New England Eye Center, Tufts Medical Center, Tufts University School of Medicine, Boston, Massachusetts, USA

\section{Correspondence to} Dr Roger A Goldberg, New England Eye Center, Tufts Medical Center, Tufts University School of Medicine, 800 Washington St. Box 450, Boston, MA 02111, USA; rgoldberg.eyemd@gmail.com

Received 8 October 2013 Revised 14 January 2014 Accepted 17 February 2014 Published Online First 13 March 2014

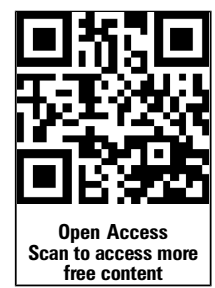

CrossMark

To cite: Goldberg RA, Waheed NK, Duker JS. Br J Ophthalmol 2014;98: ii20-ii23.

\begin{abstract}
Optical coherence tomography (OCT) imaging plays an essential role in the diagnosis and treatment of macular diseases, including those of the vitreomacular interface. OCT enables accurate diagnosis and differentiation of full thickness macular hole, lamellar macular hole and epiretinal membrane, with or without the presence of vitreomacular adhesion. This information enables earlier diagnosis and treatment when necessary, and can guide the choice of therapy. OCT is useful to facilitate discussions with patients and manage the visual expectations. Postoperatively, OCT can be helpful to optimise patient comfort and visual outcomes. As the technology continues to improve, OCT will become increasingly critical for all aspects of care for patients with macular hole and epiretinal membrane.
\end{abstract}

Since Puliafito et al first described optical coherence tomography (OCT) imaging of the macula in $1995,{ }^{1}$ OCT has evolved to become the gold standard for the diagnosis, management and follow-up of many vitreoretinal diseases. OCT-guided treatment for chronic conditions like age-related macular degeneration and macular oedema has become a de facto standard of care. Similarly, OCT has an increasingly important role in the management of diseases of the vitreomacular interface (VMI)-including full thickness macular hole (FTMH), lamellar macular hole (LMH), epiretinal membrane (ERM), and vitreomacular traction (VMT). A new classification system for diseases of the VMI relying entirely on OCT data to classify FTMH subtypes, supplements the pre-OCT Gass staging system. ${ }^{2} 3$

OCT allows for accurate diagnosis and differentiation early in the disease course and assists in preoperative decision making and planning. Similarly, OCT's role in postoperative management also helps optimise visual outcomes. This paper reviews the role of OCT in the preoperative and postoperative management of patients with FTMH and ERM.

\section{PREOPERATIVE OCT}

\section{Diagnostic utility}

Even when clinically apparent at the slit lamp, obtaining an OCT image at the time of diagnosis in patients with FTMH or ERM helps in staging, surgical planning and prognostication of anatomical and visual success. First, the OCT can confirm the diagnosis. For example, a FTMH must be distinguished from entities that can cause macular pseudohole, such as ERM, VMT or LMH. Patients with pseudoholes often retain good vision without risk of progressive photoreceptor damage, and can be more safely observed. OCT is now considered the gold standard for diagnostic confirmation of FTMH, surpassing clinical tests such as the Watzke-Allen slit beam test, in accuracy. ${ }^{4}$

Similarly, in evaluating for ERM and VMT, OCT has been demonstrated to be superior to clinical exam. In a prospective study, Do et al asked six vitreoretinal specialists to examine patients with slit lamp biomicroscopy and determine whether ERM or VMT was present, and to make a determination as to whether surgery was indicated. ${ }^{5}$ These patients then received a time domain (TD) OCT (Stratus, Zeiss). The TD OCT was found to be more sensitive than clinical exam overall, and particularly for VMT cases. ${ }^{5}$ OCT was also able to more consistently detect the presence of macular oedema, which has been corroborated in other diseases. $^{6}$

\section{Prognostic indicators}

In addition to confirming the diagnosis, OCT can be helpful in discussions with patients and their families about the disease, treatment options and visual potential after successful macular hole $(\mathrm{MH})$ or ERM surgery.

In patients with $\mathrm{MH}$, OCT can be used to accurately measure the size of the hole, which is done by using the calliper function found on most spectral domain (SD) OCT platforms to draw a horizontal line connecting the two closest components of the retina (minimum linear dimension; figure 1 ). Other measurements such as the base diameter, macular hole inner opening, as well as derived measurements such as the macular hole index, have also been studied. The minimum linear dimension is a critical factor in determining the anatomical success rate of hole closure after surgery, and forms the basis for the new international OCT-based classification system for macular holes. ${ }^{3}$ 7-9

Similarly, in patients with ERM, the integrity of the photoreceptor inner segment/outer segment (IS/OS) junction line has proven to be an important indicator of postoperative visual acuity (figure 2). Using TD OCT (Stratus), Suh et al ${ }^{10}$ found that eyes with disruption of IS/OS junction had significantly lower postoperative best corrected visual acuity (BCVA) and BCVA differences between before and after surgery than those without disruption on preoperative OCT. Falkner-Radler et al performed a similar study using TD OCT and SD OCT, and confirmed that baseline IS/OS integrity was helpful to predict the functional outcomes after surgery. ${ }^{11}$ They noted that SD OCT allowed 


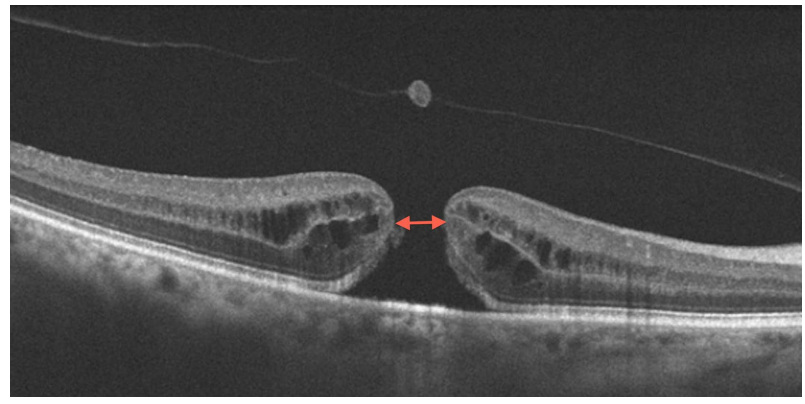

Figure 1 Full thickness macular hole (FTMH). Optical coherence tomography (OCT) shows FTMH. The calliper functions on the OCT platform can be used to measure the size of the hole; this one would be considered small ( $<250$ microns). The minimum linear dimension is indicated by the double arrowhead.

for a more precise evaluation of the IS/OS junction line and differentiation of the ERM from the retinal surface. ${ }^{11}$

The highest quality study investigating the IS/OS junction line was performed by Inoue et al. ${ }^{12}$ They prospectively divided 45 eyes of 45 patients based on the preoperative IS/OS junction: intact versus disrupted. They then analysed changes postoperatively in these two cohorts, and found that the intact IS/OS cohort had better final visual acuity and a better improvement in visual acuity. In fact, none of the 11 eyes in the disrupted IS/ OS cohort ever had a normal appearing IS/OS junction at any time point up to 1 year postoperatively. This study used SD OCT, and indocyanine green (ICG)-assisted internal limiting membrane (ILM) peeling was performed in every patient. This study also did not find a significant correlation between preoperative central foveal thickness, presence of a macular pseudohole or presence of retinal cysts with the postoperative visual acuity. $^{13}$

In patients with $\mathrm{MH}$ and ERM, a preoperative OCT can be used to assess for other conditions that may limit postoperative best-corrected visual acuity even after successful surgery, such as underlying age-related macular degeneration. This can help inform the discussion with patients and enable them to make more informed decisions regarding surgery.

\section{Surgical planning}

Additionally, with the recent availability of ocriplasmin (Jetrea, Thrombogenics) for the treatment of VMT, understanding the nature and extent of the adhesion as delineated on OCT can

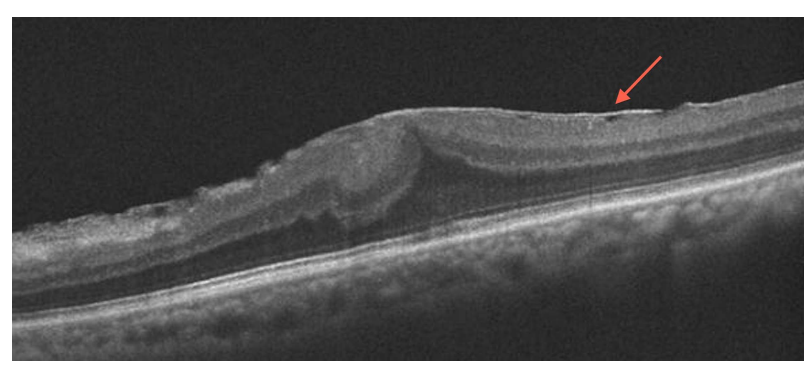

Figure 2 Epiretinal membrane. Optical coherence tomography (OCT) shows an epiretinal membrane (arrow) with intact outer retinal layers, a good prognostic sign for the postsurgical visual outcome. Additionally, this OCT highlights an area temporally where the membrane is elevated above the retina, an area where it may be surgically easier to initiate the membrane peel. guide decision-making. In the pivotal studies, one injection of ocriplasmin was able to close $40 \%$ of FTMHs smaller than 400 microns in diameter, compared with $10.6 \%$ of placebo-injected eyes. ${ }^{14}$ In FTMHs smaller than 250 microns, the anatomical closure rate with ocriplasmin was 58\%. However, in the presence of co-existent ERM, fewer than $10 \%$ of patients had resolution of their vitreomacular adhesion. Thus ocriplasmin may be a viable alternative to surgery in small and medium sized holes with persistent VMT but no ERM on OCT.

In patients who do require surgery, the risks and benefits of peeling the ILM is debated among vitreoretinal specialists in ERM and FTMH cases. In FTMH cases, studies suggest that ILM removal may improve the success rate of hole closure. However, holes smaller than 400 microns in diameter have an equivalently high rate of closure (over 90\%) whether ILM is peeled or not. ${ }^{7}$ This finding that 400 micron aperture size was important for anatomical success rate was corroborated by the ocriplasmin data, as ocriplasmin cleaves laminin and fibronectin at the vitreoretinal interface, but does not impact the ILM.

In patients with ERM, some surgeons advocate peeling ILM to prevent ERM recurrence. Some studies suggest a recurrence rate as high as $50 \%$ when the ILM is left in place, whereas the recurrence rate of ERM is $<10 \%$ when the ILM is removed. ${ }^{15} 16$ The ILM may serve as a platform for residual glial cells, hyalocytes and myofibroblasts to proliferate and re-form another ERM. Despite this, concern has arisen regarding the effect of ILM peeling on visual acuity, contrast sensitivity and the creation of microscotomas. ${ }^{17} 18$ In a single-surgeon study comparing patients who underwent ERM peel alone versus those that underwent ERM plus ILM peel, Chang et al found that there was no difference in the postoperative visual acuity, despite a greater proportion in the ERM peel only group having residual ERM present. ${ }^{19}$

Sometimes, regardless of the surgeon's intent preoperatively, the ILM comes off with the ERM simultaneously. Seidel et al examined what factors on preoperative SD OCT were predictive of residual ILM after ERM peel. ${ }^{20}$ Residual ILM was determined intraoperatively by using ICG staining after the ERM peel. They found that persistent ILM was present in $50 \%$ of cases. Not surprisingly, they found that the factors most predictive of ILM persistence after ERM peel were a greater extent of ERM elevation (a looser connection between the ERM and the retinal surface) and thicker ERMs. This may help surgeons determine beforehand which patients may warrant a second staining with ICG.

Often, the most challenging aspect of membrane peeling is initiating the flap edge. Preoperative OCT can help guide surgeons where best to initiate the membrane peel. Areas on the preoperative OCT where the ERM is elevated above the retina are easier to initiate a peel, whereas membranes with closer adherence to the retinal surface are more difficult to separate from the underling retina (figure 2). ${ }^{21}$ Similarly, OCT can be used to determine the thickest part of the membrane, as well as an identifiable edge of the membrane. ${ }^{22}$

\section{POSTOPERATIVE OCT}

\section{Guiding prone positioning}

Vitreoretinal surgeons often debate to what extent-if at all-prone positioning is needed postoperatively to maximise the rate the of hole closure after FTMH surgery. Anatomical success rates of $>90 \%$ are now routinely reported in surgical series of FTMH, leading some to question the value of facedown positioning. Several groups have demonstrated that obtaining OCT scans through gas-filled eyes is feasible, though 
scan quality can vary considerably. ${ }^{23-25}$ This requires the use of an OCT system using the macular cube scan feature to ensure that B scans through the fovea are obtained. These scans enable OCT-guided positioning postoperatively.

In a study by Shah et al, ${ }^{26} 32$ eyes underwent FTMH repair. Patients were instructed to maintain a facedown position overnight, and every patient received an OCT scan on postoperative day 1. If the OCT demonstrated a closed hole, patients were instructed to remain facedown for two additional days. If the hole was open or indeterminate, they were instructed to remain facedown for 1 week. Three-quarters of eyes had closed holes on postoperative day 1 , and of these, $96 \%$ remained closed. Of the eight eyes with open or indeterminate holes on day 1 , all were $>400$ microns in diameter preoperatively, and six had closed by postoperative week 1 . Using this algorithm of OCT-guided prone positioning, the authors achieved an overall single-procedure closure rate of $90.6 \%$.

Similarly, Masuyama et al followed patients daily with postoperative OCT. ${ }^{24}$ As soon as the hole was closed on OCT, prone positioning was stopped. With this algorithm, 16 of 16 FTMHs closed by postoperative month 1 . The authors suggest that as soon as the hole is closed on OCT, prone positioning is no longer needed. Of course, these studies do not address whether facedown positioning is necessary at all, ${ }^{27} 28$ but offer the opportunity for surgeons who do recommend prone posturing to tailor their recommendations based on OCT evidence.

\section{Explaining visual outcomes}

Postoperative OCT can also be helpful in determining why some patients have poor visual outcomes despite successful surgery. For example, OCT can easily detect subretinal or intraretinal fluid that can develop after macular surgery (figure 3). OCT can help identify this fluid, and help monitor response to therapy. Treatment options for postoperative cystoid macular oedema include topical non-steroidal anti-inflammatory drugs and corticosteroids, sub-Tenon's or intravitreal steroids or a dexamethasone intravitreal implant.

Additionally, disruptions to the retinal pigment epithelium and photoreceptors can correlate with visual outcomes. Dissociated nerve fibre layer, recurrent ERM, and photoreceptor and retinal pigment epithelium disruptions have all been reported after surgery for FTMH and ERM. ${ }^{29}$

At this point, it is not clear if one single parameter or time point on SD OCT correlates best with visual acuity. Itoh et al reviewed 51 patients with surgically closed macular holes, and correlated the length of the foveal cone outer segment tips

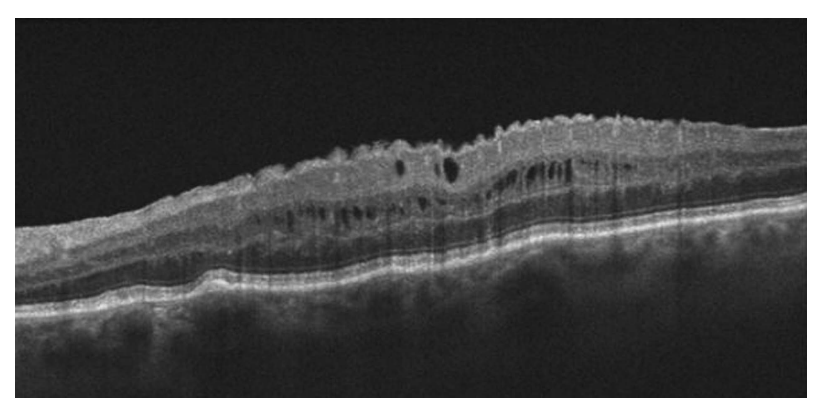

Figure 3 Postoperative macular oedema. This optical coherence tomography shows cystic intraretinal fluid after vitrectomy and membrane peel. The patient was treated with topical steroid and non-steroidal anti-inflammatory eye drops. The intraretinal fluid resolved and the visual acuity improved.
(COST) as measured by SD OCT with the best-corrected visual acuity at postoperative months $1,3,6,9$ and 12 . They found that the length of the COST line defect correlated significantly with BCVA at all time points, and that the COST line defect was gradually restored in a centripetal fashion during the postoperative period.

A similar study by Kao et $a l^{31}$ examining 77 eyes with closed macular holes found that an intact external limiting membrane at postoperative month 1 after surgery correlated best with visual outcome, regardless of the photoreceptor IS/OS status at that time. In contrast, Oh et al found in 23 patients after macular hole surgery that poorer postoperative vision did correlate with the size of the IS/OS defect, as measured by linear (raster) and composite (partial fundus image) scans. ${ }^{32}$

\section{FUTURE DIRECTIONS}

New software updates to SD OCT systems now enable en face imaging of the retinal surface. Rispoli et $a l^{33}$ examined 20 consecutive patients with idiopathic ERMs using en face OCT imaging, and identified areas of small craters and pits adjacent to the plaque membrane. They hypothesise that membrane contraction causes localised tearing and folding of the ILM, exposing the bare retinal nerve fibre layer underneath. These would not be good places to initiate membrane peel during surgery. Postoperatively, the authors identified areas of dimpling on en face imaging that they speculate are consistent with previously described reports of dissociated optic nerve fibre layer, though they suggest that they may be Muller cells regenerating their end feet. How en face imaging may be useful is yet to be determined.

Intraoperative OCT offers another exciting aid to the vitreoretinal surgeon during FTMH and ERM surgeries. Though no real-time, integrated system yet exists to provide the surgeon with live feedback of the precise layers being grasped, active research in this area is being pursued. ${ }^{34}$

Finally, swept source (SS) OCT imaging may further add to our understanding of $\mathrm{MH}$ and ERM pathophysiology and surgical outcomes. SS OCT can image at speeds of 100 000-400 000 axial scans per second with a $1050 \mathrm{~nm}$ light source, enabling wide-field imaging, improved deep choroidal imaging and faster acquisition speeds. ${ }^{35}$ What role SS OCT will play for patients with ERM and $\mathrm{MH}$ is yet to be determined.

\section{CONCLUSION}

OCT has revolutionised all facets of our management of patients with FTMH and ERM. Over the past decade, OCT has become an essential component of the preoperative evaluation of FTMH and ERM, confirming the diagnosis, aiding in surgical planning and explaining visually to patients what these diagnoses mean. Similarly, postoperatively, OCT can help predict and explain the visual outcome, guide therapeutic decision-making such as prone positioning or the need for-and response to-treatment for cystoid macular oedema. New advances currently in development will likely expand the role of OCT even further, for the benefit of our patients with FTMH and ERM.

Contributors All authors contributed to the (1) conception and design of this manuscript, (2) its drafting and revisions, and (3) its final approval.

Competing interests JSD receives research support from Carl Zeiss Meditech and Optovue.

Provenance and peer review Not commissioned; externally peer reviewed.

Open Access This is an Open Access article distributed in accordance with the Creative Commons Attribution Non Commercial (CC BY-NC 3.0) license, which permits others to distribute, remix, adapt, build upon this work non-commercially, 
and license their derivative works on different terms, provided the original work is properly cited and the use is non-commercial. See: http://creativecommons.org/ licenses/by-nc/3.0/

\section{REFERENCES}

1 Puliafito CA, Hee MR, Lin CP, et al. Imaging of macular diseases with optical coherence tomography. Ophthalmol 1995;102:217-29.

2 Johnson RN, Gass JD. Idiopathic macular holes. Observations, stages of formation, and implications for surgical intervention. Ophthalmol 1988;95:917-24.

3 Duker JS, Kaiser PK, Binder S, et al. The International Vitreomacular Traction. Study Group Classification of Vitreomacular Adhesion, Traction, and Macular Hole. Ophthalmol 2013;120:2611-9.

4 Tanner V, Williamson TH. Watzke-Allen slit beam test in macular holes confirmed by optical coherence tomography. Arch Ophthalmol 2000;118:1059-63.

5 Do DV, Cho M, Nguyen QD, et al. The impact of optical coherence tomography on surgical decision making in epiretinal membrane and vitreomacular traction. Trans Am Ophthalmol Soc 2006;104:161-6.

6 Browning DJ, McOwen MD, Bowen RM Jr, et al. Comparison of the clinical diagnosis of diabetic macular edema with diagnosis by optical coherence tomography. Ophthalmol 2004;111:712-5.

7 Tadayoni R, Gaudric A, Haouchine B, et al. Relationship between macular hole size and the potential benefit of internal limiting membrane peeling. $\mathrm{Br} J$ Ophthalmol 2006:90:1239-41.

8 Ulrich S, Haritoglou C, Gass C, et al. Macular hole size as a prognostic factor in macular hole surgery. Br I Ophthalmol 2002;86:728-35.

9 Ip MS, Baker BJ, Duker JS, et al. Anatomical outcomes of surgery for idiopathic macular hole as determined by optical coherence tomography. Arch Ophthalmol 2002;1:2029-35

10 Suh MH, Seo JM, Park KH, et al. Associations between macular findings by optical coherence tomography and visual outcomes after epiretinal membrane removal. $A m$ J Ophthalmol 2009;147:473-80.

11 Falkner-Radler $\mathrm{Cl}$, Glittenberg $\mathrm{C}$, Hagen $\mathrm{S}$, et al. Spectral-domain optical coherence tomography for monitoring epiretinal membrane surgery. Ophthalmol 2010;117:798-805.

12 Inoue M, Morita S, Watanabe $\mathrm{Y}$, et al. Inner segment/outer segment junction assessed by spectral-domain optical coherence tomography in patients with idiopathic epiretinal membrane. Am J Ophthalmol 2010;150:834-9.

13 Inoue M, Morita S, Watanabe $\mathrm{Y}$, et al. Preoperative inner segment/outer segment junction in spectral-domain optical coherence tomography as a prognostic factor in epiretinal membrane surgery. Retina 2011;31:1366-72.

14 Stalmans P, Benz MS, Gandorfer A, et al. Enzymatic vitreolysis with ocriplasmin for vitreomacular traction and macular holes. N Engl J Med 2012;367:606-15.

15 Grewing R, Mester U. Results of surgery for epiretinal membranes and their recurrences. Br J Ophthalmol 1996;80:323-6.

16 Park DW, Dugel PU, Garda J, et al. Macular pucker removal with and without internal limiting membrane peeling: pilot study. Ophthalmol 2003;110:62-4.

17 Bovey EH, Uffer S, Achache F. Surgery for epimacular membrane: impact of retinal internal limiting membrane removal on functional outcome. Retina 2004;24:728-35.
18 Kwok A, Lai TY, Yuen KS. Epiretinal membrane surgery with or without internal limiting membrane peeling. Clin Experiment Ophthalmol 2005;33:379-85

19 Chang S, Gregory-Roberts EM, Park $S$, et al. Double peeling during vitrectomy for macular pucker. JAMA Ophthalmol 2013;131:525-30.

20 Seidel G, Weger M, Stadlmuller L, et al. Association of preoperative optical coherence tomography markers with residual inner limiting membrane in epiretinal membrane peeling. PLOS ONE 2013;8:e66217.

21 Kim JS, Chhablani J, Chan CK, et al. Retinal adherence and fibrillary surface changes correlate with surgical difficulty of epiretinal membrane removal. Am J Ophthalmol 2012;153:692-7.

22 Hirano Y, Yasukawa T, Ogura Y. Optical coherence tomography guided peeling of macular epiretinal membrane. Clin Ophthalmol 2010:5:27-9.

23 Sato H, Kawasaki R, Yamashita H. Observation of idiopathic full-thickness macular hole closure in early postoperative period as evaluated by optical coherence tomography. Am J Ophthalmol 2003;136:185-7.

24 Masuyama K, Yamakiri K, Arimura N, et al. Posturing time after macular hole surgery modified by optical coherence tomography images: a pilot study. Am J Ophthalmol 2009;147:481-8.

25 Ehlers JP, Yuan A, Kaiser PK, et al. Trans-tamponade optical coherence tomography: postoperative imaging in gas-filled eyes. Retina 2013;33 $1172-8$

26 Shah SP, Manjunath V, Rogers AH, et al. Optical coherence tomography-guided facedown positioning for macular hole surgery. Retina 2013;33:356-62.

27 Tornambe PE, Poliner LS, Grote K. Macular hole surgery without face-down positioning. A pilot study. Retina 1997:17:179-85.

28 Tadayoni R, Vicaut E, Devin F, et al. A randomized controlled trial of alleviated positioning after small macular hole surgery. Ophthalmol 2011;118:150-5.

29 Michalewska Z, Michalewski J, Cisiecki S, et al. Correlation between foveal structure and visual outcome following macular hole surgery: a spectral optical coherence tomography study. Graefes Arch Clin Exp Ophthalmol 2008;246:823-30.

30 Itoh Y, Inoue M, Rii T, et al. Correlation between length of foveal cone outer segment tips line defect and visual acuity after macular hole closure. Ophthalmol 2012;119:1438-46.

31 Kao TY, Yang CM, Yeh PT, et al. The value of combining autofluorescence and optical coherence tomography in predicting the visual prognosis of sealed macular holes. Am J Ophthalmol 2013:156:149-56.

32 Oh J, Smiddy WE, Flynn HW Jr, et al. Photoreceptor inner/outer segment defect imaging by spectral domain OCT and visual prognosis after macular hole surgery. Invest Ophthalmol Vis Sci 2010;51:1651-8.

33 Rispoli M, Le Rouic JF, Lesnoni G, et al. Retinal surface en face optical coherence tomography: a new imaging approach in epiretinal membrane surgery. Retina 2012;32:2070-6

34 Hahn P, Migacz J, O'Connell R, et al. The use of optical coherence tomography in intraoperative ophthalmic imaging. Ophthalmic Surg Lasers Imaging 2011;42: S85-94.

35 Potsaid B, Baumann B, Huang D, et al. Ultrahigh speed $1050 \mathrm{~nm}$ swept source Fourier domain OCT retinal and anterior segment imaging at 100,000 to 400000 axial scans per second. Opt Express 2010;18:20029-48. 